\title{
SOP 07: Data Flow, Monitoring, and Archiving
}

\section{Background}

SOP 07 outlines the data flow among the parties involved in the conduct of a phase I or phase II or phase III trial. The procedures specified in this SOP primarily concern the documentation of results on the Case Report Forms (CRFs), their verification by the monitor of the study, the entry of the data into the computerized data base and their validation at the data center of the CESAR.

The flow of information as specified below and the verification of the study data for each individual patient by the monitor of the sponsor aims at ensuring that strict standards regarding data quality are implemented for all studies performed by CESAR.

\section{Patient Registration}

As a rule, the eligibility of a patient for entering a phase I or phase II or phase III study should be verified by an independent assessor before the patient receives the first dose of the study medication. Each study protocol must specify the reporting lines, i.e. the role of CESAR's data center and of the sponsor's study monitor, as well as any registration forms to be used. The general procedure is as follows:

- The investigator performs all screening investigations and compares the patient characteristics with the inclusion and exclusion criteria of the protocol. If the investigator considers a patient eligible for the study, he will transmit the relevant information to data center or the monitor.

- The data center or the monitor will immediately check the entries in this form against the eligibility check data. Any discrepancies that may affect the patient's eligibility for the study will be jointly clarified by the sponsor and the coordinating investigator. Any decision will be documented in writing.

\section{CRF Submission Paths}

CRF submission paths are depicted in figure 1.

\section{Notes Regarding Data Submission Paths}

- Direct entry of data into a computerized documentation system by investigators is acceptable if the procedures are agreed upon with the Data Center of CESAR.

- Each addition to or modification of a CRF must be dated and initialled by the investigator. Changes must be made in a way which does not obscure the original entry (use of masking fluids is not allowed); if required, the reason for the correction must be given.

- A written query may be issued to resolve any data discrepancy. This will allow for changes without the risks associated with repeated mailing of original documents.

\section{Trial Documentation}

- Study monitoring is the responsibility of the monitor of the sponsor and follows the procedures defined in the sponsor's own SOP.

- The monitor of the sponsor is responsible for the timely transmission of the CRFs to the data center of the CESAR.

- The monitor of the sponsor makes sure that queries and requests for additions to or corrections of the CRFs are promptly (within 4 weeks) attended to and dealt with according to the data submission paths described in section 8.2 of ICH-GCP [1].

At the end of one reporting interval (typically one treatment cycle), the investigator has 4 weeks for submission of the completed CRFs to the monitor, unless otherwise specified in the trial protocol.

It is the monitor's obligation to report to the Coordinating Investigator (CI) the failure of an investigator to comply with the timely completion of CRFs. 
1. Completion of the CRFs by the investigator and/or staff member designated by the investigator

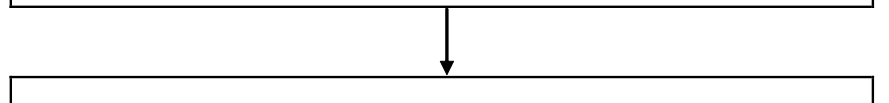

2. Signing of the CRFs by the investigator

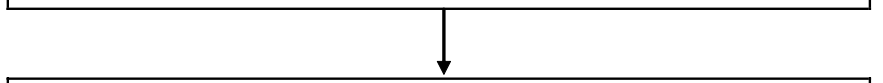

3. Submission (in person/by mail) of the original CRFs to the monitor of the sponsor by the investigator

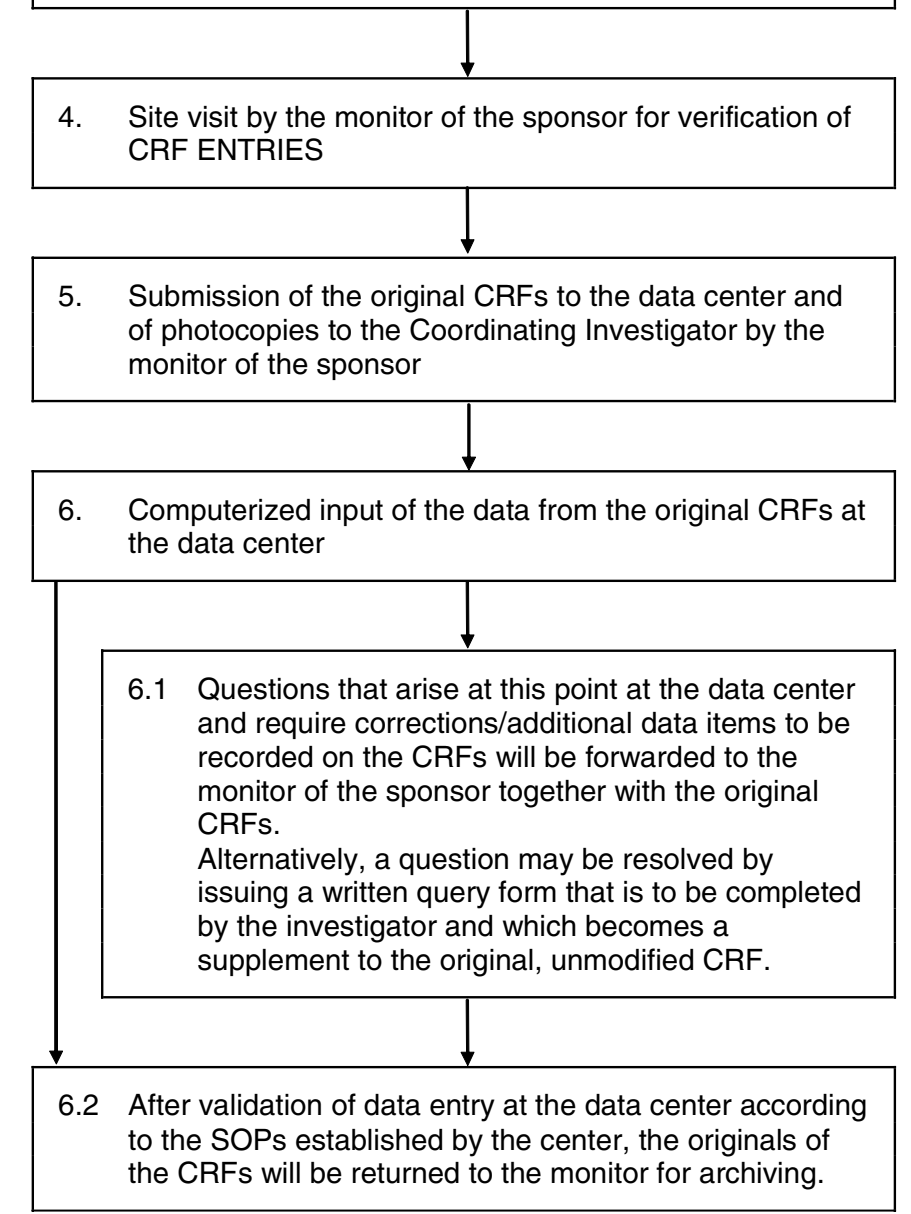

Fig. 1. CRF submission paths.

\section{Site Visit Report}

The monitor will write a site visit report after each site visit and summarize the relevant findings, specifically any action items, in a letter to the clinical investigator, with a copy to the CI.

\section{Archiving of Essential Documents}

Archiving of documents relating to a clinical trial must comply with EEC guidelines [2]. Refer to section 8 of ICH-GCP for a complete list of 'essential documents' and the assignment of respective archiving obligations to investigator and/or sponsor.

- The monitor is responsible for archiving at the sponsor's institution of the originals of all completed CRFs from the clinical trial. Each principal investigator should retain in the study files copies of those CRFs that were completed under his/her responsibility.

- The investigator shall archive a copy of the approval by the Ethics Committee, his/her patient identification list, the originals of the signed informed consent forms, the patient files and other source documents.

- All source documents and trial-related documents must be made available for inspection at the request of relevant authorities. The documents must be archived for the minimum period required by the institution, but not less than 15 years. No original of a trial-related document should be disposed without prior written agreement by the sponsor.

\section{References}

1 ICH-GCP: EMEA: Note for Guidance on Good Clinical Practice (CPMP/ICH/135/95).

2 Guideline 91/507/EEC, as revised July 19, 1991. 